\title{
EMG-informed neuromusculoskeletal models accurately predict knee loading measured using instrumented implants
}

This paper was downloaded from TechRxiv (https://www.techrxiv.org).

LICENSE

CC BY 4.0

SUBMISSION DATE / POSTED DATE

$15-09-2021$ / 23-09-2021

CITATION

Bennett, Kieran; Pizzolato, Claudio; Martelli, Saulo; Bahl, Jasvir; Sivakumar, Arjun; Atkins, Gerald; et al. (2021): EMG-informed neuromusculoskeletal models accurately predict knee loading measured using instrumented implants. TechRxiv. Preprint. https://doi.org/10.36227/techrxiv.16620574.v1

$\mathrm{DOI}$

10.36227/techrxiv.16620574.v1 


\title{
EMG-informed neuromusculoskeletal models accurately predict knee loading measured using instrumented implants
}

\author{
Kieran J. Bennett, Claudio Pizzolato, Saulo Martelli, Jasvir S. Bahl, Arjun Sivakumar, Gerald J. \\ Atkins, L. Bogdan Solomon, Dominic Thewlis
}

\begin{abstract}
Objective: Using a musculoskeletal modelling framework, we aimed to (1) estimate knee joint loading using static optimization (SO); (2) explore different calibration functions in electromyogram (EMG)-informed models used in estimating knee load; and (3) determine, using an EMG-informed stochastic method, if the variation in knee loads could be predicted by only investigating the uncertainty in muscle forces. Methods: Musculoskeletal models for three individuals with instrumented knee replacements were generated. Muscle forces were calculated using SO, EMG-informed, and EMG-informed stochastic methods. Measured knee joint loads from the prostheses were compared to the SO and EMG-informed solutions. Root mean square error (RMSE) in joint load estimation was calculated, and the muscle force ranges were compared. Results: The RMSE ranged between 192-674 N, 152-487 N, and 7-108 $\mathrm{N}$ for the SO, the calibrated EMG-informed solution, and the best fit stochastic result, respectively. The stochastic method produced solution spaces encompassing the measured joint loading up to $98 \%$ of stance. Conclusion: Uncertainty in muscle forces can account for total knee loading and it is recommended that, where possible, EMG measurements should be included to estimate knee joint loading. Significance: This work shows that the inclusion of EMGinformed modelling allows for better estimation of knee joint loading when compared to SO.
\end{abstract}

Index Terms-Biomechanics; Biomechanical simulation; neuromusculoskeletal models, electromyography

\section{INTRODUCTION}

$\mathrm{D}$ ETERMINING the internal loads acting on the knee joint is central to characterising its local mechanical environment during activity, and understanding how the environment alters with injury, disease and treatment [1]. However, measuring joint loads is only possible through instrumented joint replacements [2,3], thus limiting knowledge of joint loading to a small cohort of people worldwide. Musculoskeletal modelling can be used to estimate joint loads using experimental data, including kinematics, foot-ground reaction forces, and electromyograms (EMG). This modelling

This work was submitted on the $15^{\text {th }}$ September 2021. This research program is supported by an Australian Government Research Training Program (RTP) Scholarship. D. Thewlis receives fellowship funding from NHMRC (ID: 1126229), and S. Martelli receives funding from the ARC (DP180103146; FT180100338; IC190100020).

K.J. Bennett, A. Sivakumar, J.S. Bahl, G.J. Atkins, L.B. Solomon, and D. Thewlis are with the Centre for Orthopaedic and Trauma Research, The framework estimates the muscle forces required to ensure joint equilibrium during motion [4-6].

The human musculoskeletal system is highly redundant, meaning there are more muscles than mechanical degrees of freedom. This implies that there is an infinite number of muscle activation solutions to produce the same observed motion [7-9]. Several methods have been described to estimate muscle forces, including: static optimization (SO), which assumes that the nervous systems use an a priori optimal strategy for the control of muscles [6, 10, 11]; EMG-informed methods, which constrain the muscle activation using the experimentally measured muscle electrical activity in selected superficial muscles[12-14]; and stochastic methods, which generate a spectrum of muscle forces and joint loadings consistent with the model employed and uncertainties associated with model parameters $[7,15]$.

EMG-informed methods can model individual muscle activation [14, 16-19], accounting for co-contractions [14], but require parameter calibration due to the nonlinear relationships between EMG measures and muscle function [5, 13]. In calibration, physiological neuromuscular parameters are adjusted to minimize an objective function. Several objective functions have previously been implemented to generate physiologically plausible joint contact forces $[14,20]$, and validated for one individual but their generalizability has not been evaluated [17]. It is currently unclear if there exists an optimal calibration function which could be used for multiple subjects to model muscle combinations while yielding accurate estimation of joint loading.

Stochastic methods can explore the variability in muscle contributions to motion by sampling the space of muscle forces satisfying joint equilibrium within prescribed force constraints $[15,21]$. For example, the uncertainty in muscle forces (due to a lack of known muscle forces) can be modelled by constraining them to within their physiological boundaries (i.e., between zero and their peak force). Uncertainty in measured parameters (i.e., EMG measurements, body position, and ground reaction forces) can also be investigated in this framework to statistically model movement. Van Veen and co-workers demonstrated that

University of Adelaide, Adelaide, South Australia, Australia. C. Pizzolato is with Menzies Health Institute - Griffith Centre of Biomedical and Rehabilitation Engineering, Griffith University, Gold Coast, Queensland, Australia. S. Martelli is with the School of Mechanical Medical \& Process Engineering, Queensland University of Technology, Brisbane, Queensland, Australia. (Correspondence e-mail: kieran.bennett@ adelaide.edu.au). 
there exists a muscle coordination which generates the measured knee load in the model of one volunteer while walking [21]. However, there were discrepancies between stochastic perturbations of a SO solution, the measured knee load, and the related muscle coordination. Investigating the uncertainty in muscle force predictions, and modelling them as probabilistic variables, might enable determining if there exists a set of muscle forces that result in the measured joint loading.

The aims of this study were to: (1) estimate knee loading using SO; (2) explore different calibration functions in EMGinformed models used in estimating knee load; and (3) determine, using an EMG-informed stochastic method, if the variation in knee loads can be predicted by only investigating the uncertainty in muscle forces. We hypothesize that (1) using a single optimization function to calibrate EMG-informed models, accurate estimates of joint loading can be obtained for multiple individuals and (2) the knee load will be contained within the range of solutions in the stochastic solution. All estimations of knee load, including their directional components, will be compared to the loads measured in vivo.

\section{METHODS}

\section{A. Data}

Data were obtained from the Grand Challenge Competition to Predict In Vivo Knee Loads [2], specifically, those for challenges four, five, and six. These were chosen due to their file format (challenges three onwards present data in .c3d) and volume of walking data available (challenge three had four useable trials, while challenges four and five had five useable trials, and challenge six had six useable trials). Each challenge includes a comprehensive dataset from a single participant with an instrumented knee replacement. The participants in challenges five and six both had the second generation of the eTibia implant, which measured directional loading as individual components.

\section{B. Preprocessing and EMG}

The marker trajectories, ground reaction forces, and EMG data from one static trial and five over ground walking gait trials were used for each of the three participants. Marker trajectories and ground reaction forces were low pass filtered with a cutoff frequency of $8 \mathrm{~Hz}$, and EMG signals were band-pass filtered to 30-300 $\mathrm{Hz}$ [5], full wave rectified, low pass filtered with a cutoff frequency of $6 \mathrm{~Hz}$, and subsequently amplitude normalized to the maximum value across all trials [5, 22]. All filtering was completed using $2^{\text {nd }}$ order zero lag Butterworth filters.

Between 10 and 11 EMG signals were used for each challenge (Table 1, processed signals in Supplementary Material). EMG signals were not used if the muscles were weak (model maximum isometric force less than 500 N. i.e. tensor fascia latae, and sartorius), if the signal was too noisy (as determined by a single operator via visual inspection, considering the raw EMG signals), or if the inclusion of the measurements produced spurious results in the EMG-informed solution (e.g. soleus in challenges 4 and 5 were estimated to activate to over $50 \%$ because it was the most 'efficient' muscle to drive the ankle plantarflexion).
Table 1 Muscles informed by EMG in the EMG-informed and stochastic models for each challenge.

\begin{tabular}{r|ccc} 
& \multicolumn{4}{|c}{ Challenge } \\
Muscle & 4 & 5 & 6 \\
\hline Vastus lateralis & $\checkmark$ & $\checkmark$ & $\checkmark$ \\
Vastus medialis & $\checkmark$ & $\checkmark$ & Noise \\
Tibialis anterior & $\checkmark$ & $\checkmark$ & $\checkmark$ \\
Biceps femoris long head & $\checkmark$ & $\checkmark$ & Noise \\
Semimembranosus & $\checkmark$ & $\checkmark$ & $\checkmark$ \\
Lateral gastrocnemius & $\checkmark$ & $\checkmark$ & $\checkmark$ \\
Medial gastrocnemius & $\checkmark$ & $\checkmark$ & $\checkmark$ \\
Gluteus maximus & $\checkmark$ & Noise & $\checkmark$ \\
Gluteus medius & Noise & Noise & Noise \\
Adductor magnus & $\checkmark$ & $\checkmark$ & $\checkmark$ \\
Rectus femoris & $\checkmark$ & $\checkmark$ & $\checkmark$ \\
Peroneus longus & $\checkmark$ & $\checkmark$ & $\checkmark$ \\
Soleus & Produced Spurious Results & $\checkmark$
\end{tabular}

\section{Musculoskeletal Models}

The OpenSim lower limb model (Gait2392) [11] was used as a base to tailor individual musculoskeletal models. This model has 23 degrees of freedom (DOF) and 92 musculotendon units (MTUs) [11]. The model was scaled to the mass and anthropometry of each participant using static trial marker data. The Musculoskeletal Atlas Project (MAPClient) was used to perform principal component morphing of the MAPClient pelvis, femur, and shank to the experimental markers in the static trial, whereby scale factors for the three segments were calculated as the distance between corresponding marker pairs on the two models [23]. The trunk and feet were linearly scaled, as statistical shape models for these segments are not currently implemented in the MAPClient [24]. Segment masses were determined using the mass distributions from de Leva [25], with additional mass added to the calcaneus segment, based on the shoe worn (approximately $0.3 \mathrm{~kg}$ ). The muscle and tendon lengths were optimized based on cadaveric specimens [26] using the implementation from Modenese et al. [27]. Muscle maximum isometric forces were then scaled to the participant's height and mass, using the relationships from Handsfield et al. [28], with a maximum muscle stress of $0.61 \mathrm{MPa}$ [5].

Using OpenSim (Version 3.3) [11], the inverse kinematics tool reconstructed the model motion from marker data [11]. External joint moments were calculated using the recursive Newton-Euler method in the inverse dynamics tool, and muscle moment arms, lengths, and velocities were calculated using the muscle analysis tool [11].

\section{Muscle Recruitment Models}

Three methods to determine muscle recruitment were implemented: (1) SO; (2) EMG-informed modelling, and (3) an EMG-informed stochastic method. For the EMG-informed and stochastic methods, the gait2392 model was reduced to only the implanted side.

\section{1) Static Optimization}

Muscle contributions were calculated to balance the external moments $(\tau)(1)$ by minimizing muscle activation $\left(\mathrm{a}_{\mathrm{m}}\right)$ in the objective function (2) using the static optimization tool in OpenSim [11]. Muscle activations were related to joint 
moments using the Hill type muscle model $f\left(F_{m}^{0}, l_{m}, v_{m}\right)$ multiplied by their lever arms $\left(\mathrm{r}_{\mathrm{m}, \mathrm{j}}\right)[29]$. The activation was constrained for each muscle $(\mathrm{m})$ to between zero and one [6]. The force-velocity relationships were used, and residual actuators were included with an optimal moment of $1 \mathrm{Nm}$. Joint loads were calculated using the joint reaction analysis tool.

$$
\begin{gathered}
\sum_{m=1}^{n}\left[a_{m} f\left(F_{m}^{0}, l_{m}, v_{m}\right)\right] r_{m, j}=\tau_{j} \\
f_{o b j}=\sum_{m=1}^{n}\left(a_{m}\right)^{2}
\end{gathered}
$$

\section{2) Calibrated EMG-Informed Neuromusculoskeletal Modelling Toolbox}

The EMG signals were mapped to their corresponding model MTUs (Table 2) [30]. To do this, the non-linear relationships between EMG signals and MTU activations ( $\mathrm{C} 1, \mathrm{C} 2$, and shape factor from the work of Lloyd and Besier [13], and a parameter to scale the EMG signal) were modified using the Calibrated EMG-Informed Neuromusculoskeletal Modelling Toolbox (CEINMS) calibration step [5, 12]. In this step, muscle parameters are adjusted such that a user defined objective function is minimized. The generic composite objective function (3) was applied. In this, $f_{\text {obj }}$ is the objective function, $w_{n}$ is the weighting coefficient for the function $\left(f_{n}\right)$ and $k_{n}$ is the exponent.

Table 2 Musculotendon units in the gait2392 model driven by EMG signals.

\begin{tabular}{r|l} 
EMG Signal & Gait 2392 MTU \\
Vastus lateralis & vas_lat \\
Vastus medialis & vas_med \\
Tibialis anterior & tib_ant \\
Biceps femoris long head & bifemlh \\
Semimembranosus & semimem, semiten \\
Lateral gastrocnemius & lat_gas \\
Medial gastrocnemius & med_gas \\
Gluteus maximus & glut_max $(1,2,3)$ \\
Adductor magnus & add_mag $(1,2,3)$ \\
Rectus femoris & rec_fem \\
Peroneus longus & per_long \\
Soleus & soleus \\
& \\
$f_{\text {obj }}=w_{1} f_{1}^{k_{1}}+w_{2} f_{2}^{k_{1}}+\cdots+w_{n} f_{n}^{k_{n}}$
\end{tabular}

Based on the work from Gerus et al. [17] and Hoang et al. [14], it was necessary to include model-estimated joint loading in the calibration step to minimize total muscle forces, and hence joint loads. Therefore, the objective functions investigated in this study minimized a combination of peak joint loading and normalized external moment error (4). Twenty-four different combinations of weightings and exponents were tested to determine a function which minimized root mean square error (RMSE) between the in vivo measured and estimated joint loading for all trials (functions in Supplementary material).

Joint dynamics were filtered using a low-pass $2^{\text {nd }}$ order zerolag Butterworth filter with cutoff frequency $8 \mathrm{~Hz}$. Muscle forces for each trial were estimated by driving four degrees of freedom (hip flexion, adduction; knee flexion; ankle flexion) in EMG-assisted mode [31]. For each subject, the first two trials were used for calibration, and subsequent three for analysis.

$$
\begin{gathered}
f_{\text {obj }}=w_{1} \operatorname{NRMSE}(\tau, \hat{\tau})^{k_{1}}+w_{2} F_{\text {hip }}^{\text {peak }^{k_{2}}}+ \\
w_{3} F_{\text {knee }}^{\text {peak }}{ }^{k_{3}}+w_{4} F_{\text {ankle }}^{\text {peak }} k_{4}
\end{gathered}
$$

\section{3) EMG-informed Stochastic Modelling}

The solution space of the equilibrium equation at the joints (5) was formulated using the muscle lever arms $(\bar{B})$ and the external joint moments $(\bar{\tau})$ from muscle analysis and inverse dynamics. The muscle forces $(\bar{F})$ were then sampled using the Markov-Chain Monte-Carlo method implemented in Metabolica [15]. The constraints on the muscle forces were set up in two steps. First step: muscles were bound between zero and one half of the peak muscle force, determined using the force-length-velocity Hill-type muscle model from Thelen et al. [32]. Second step: muscles that were driven in the EMGinformed approach were constrained between zero and one standard deviation above the mean muscle force estimated from CEINMS. The solution space of the equilibrium equation at the joints was sampled using one million iterations per frame and constraining external moment error to $1 \mathrm{Nm}$.

$$
\bar{B} \times \bar{F}=\bar{\tau}
$$

\section{E. Data Analysis}

Joint loadings were normalized to 101 data points across stance phase and RMSE calculated between measured and modelled knee joint loadings for both SO and EMG-informed methods. Statistical analyses were performed using non-parametric paired one-dimensional t-tests were undertaken using statistical non-parametric mapping (SnPM) in spm1d with significance set at $\alpha=0.125\left(1 / 2^{\text {nTrials }}\right)[33,34]$. The directional loading for each method was also presented for qualitative assessment. To investigate the variation in muscle control, the range muscle forces for each method were qualitatively compared.

\section{RESULTS}

Objective function number eight (6) resulted in the lowest overall RMSE among all objective functions evaluated while also not showing any force discontinuities (summary of RMSE shown in Fig. 1, Joint loadings for the three best calibration functions shown in Supplementary Material). This function compromised between minimizing the moment error and maximum model predicted joint loads. This reduced the total loading at each joint at the expense of some moment balancing (comparison of joint moments in Supplementary Material).

$$
\begin{gathered}
f_{\text {obj }}=\operatorname{NRMSE}(\tau, \hat{\tau})^{2}+ \\
0.05 \cdot\left(\max \left(F_{\text {hip }}\right)+\max \left(F_{\text {knee }}\right)+\max \left(F_{\text {ankle }}\right)\right)
\end{gathered}
$$

For challenges four and five (Fig. 2), SO, on average, overestimated the second peak loading by $717 \mathrm{~N}$ (range: 55$1432 \mathrm{~N}$ ) whereas the EMG-informed solutions overestimated the initial loading by, on average, $560 \mathrm{~N}$ (range -259-1202 N) but produced a lower error for the second peak loads (mean absolute error $216 \mathrm{~N}$, range 93-328 N). The stochastic solution 


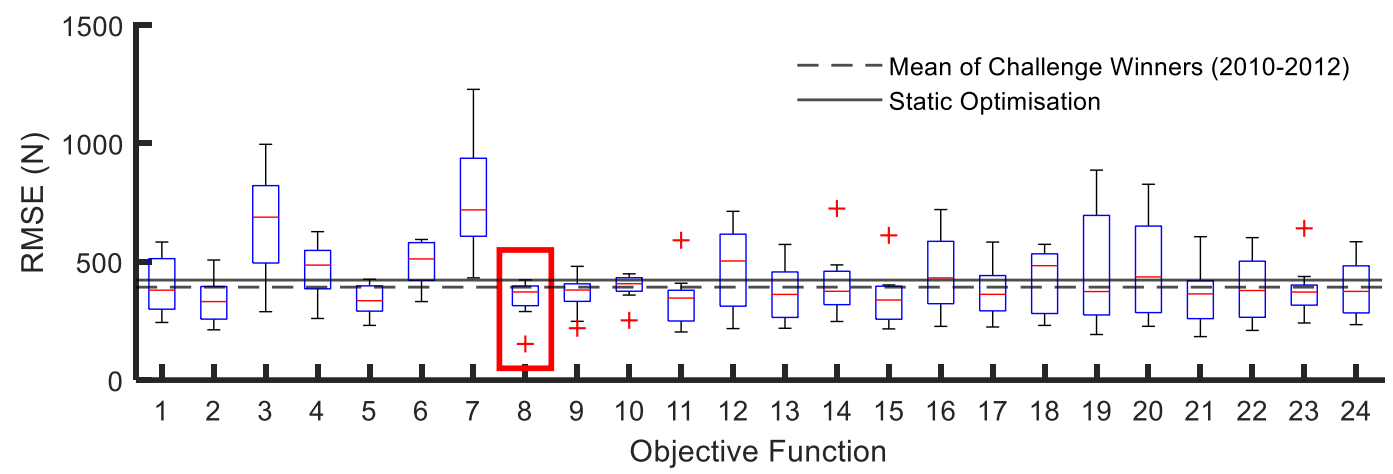

Fig. 1. Box plots showing the RMSE for each of the objective functions tested for CEINMS (summarized in Supplementary Material) with the selected calibration function highlighted. Red pluses are trials which were statistical outlier for that objective function.

space encompassed the measured loading for $76 \%$ (challenge 6 , Trial 3) to $98 \%$ (challenge 5, Trials 1,3) of stance phase.
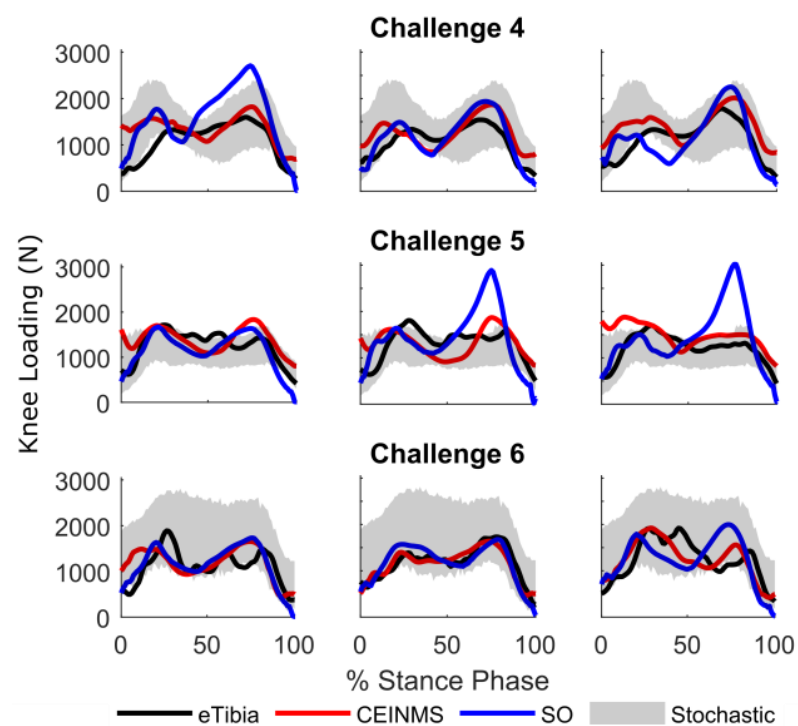

Fig. 2. Knee loads from the instrumented prostheses (eTibia, black), overlaid with the solutions from CEINMS (red), SO (blue) and the stochastic method (gray) for individual over ground walking trials.

Comparing the directional components of the total knee loading (only available for challenges 5 and 6 ) for SO and CEINMS (Fig. 3 and 4), the superior-inferior components were best estimated by the models. Considering the peak superiorinferior loading, the SO solution shows error in challenge 5 (1324-1438 N), and a comparably smaller error in challenge 6 (2-495 N). For both methods, the anterior posterior (RMSE SO: 170-250 N, CEINMS: 267-386 N) and medial-lateral (RMSE SO: 52-89 N, CEINMS: 65-112 N) loads were not well estimated. Considering the solution space of possible joint loading scenarios from the stochastic method, only the superiorinferior loading was entirely within the solution space for all trials.

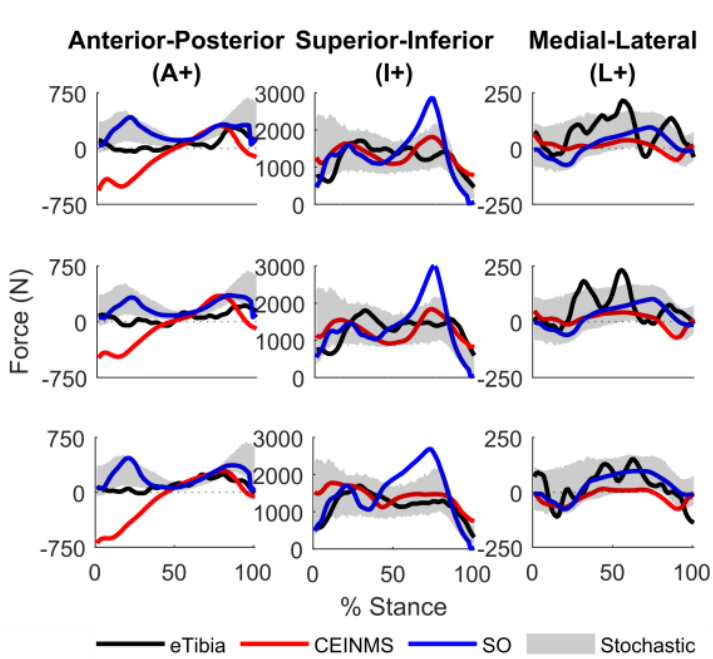

Fig. 3. Directional components of joint loading for the challenge 5 dataset from the instrumented prostheses (eTibia, black), overlaid with the solutions from SO (blue), CEINMS (red), and the stochastic method (grey) for individual over ground walking trials. Each row represents a single trial, and for each direction, anterior, inferior, and lateral are positive.

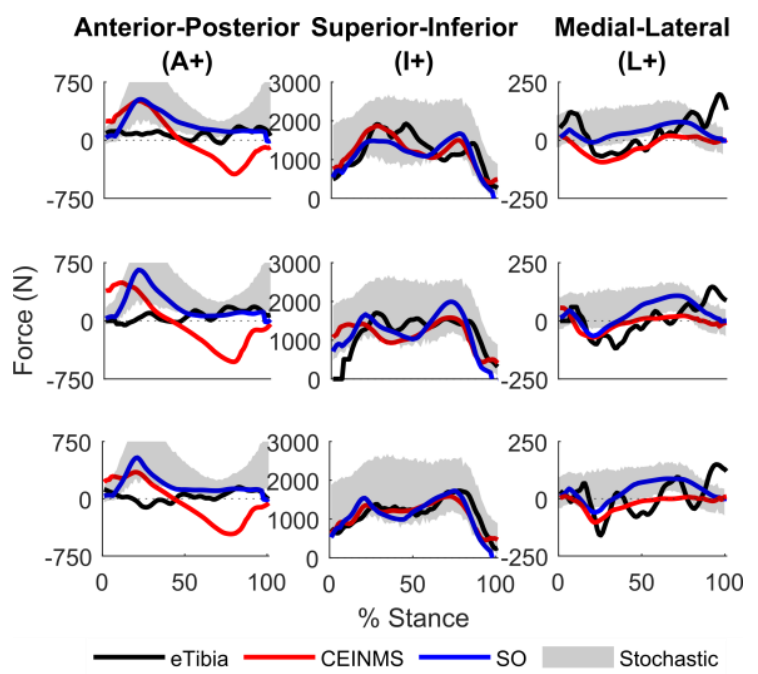

Fig. 4. Directional components of joint loading for the challenge 6 dataset from the instrumented prostheses (eTibia, black), overlaid with the solutions from SO (blue), CEINMS (red), and the stochastic method (grey) for individual over ground walking trials. Each row 
represents a single trial, and for each direction, anterior, inferior, and lateral are positive.

The SnPM analysis of the knee loads (Fig. 5) showed a series of very short supra-threshold clusters where the maximum detectable significance $(0.125)$ was exceeded. None of these clusters, however, were longer than $1 \%$ of stance phase. Across the nine trials, the mean RMSE (Fig. 6) for SO was $422 \mathrm{~N}$ (range 192-674 N) and for CEINMS was $342 \mathrm{~N}$ (range 152$487 \mathrm{~N})$.
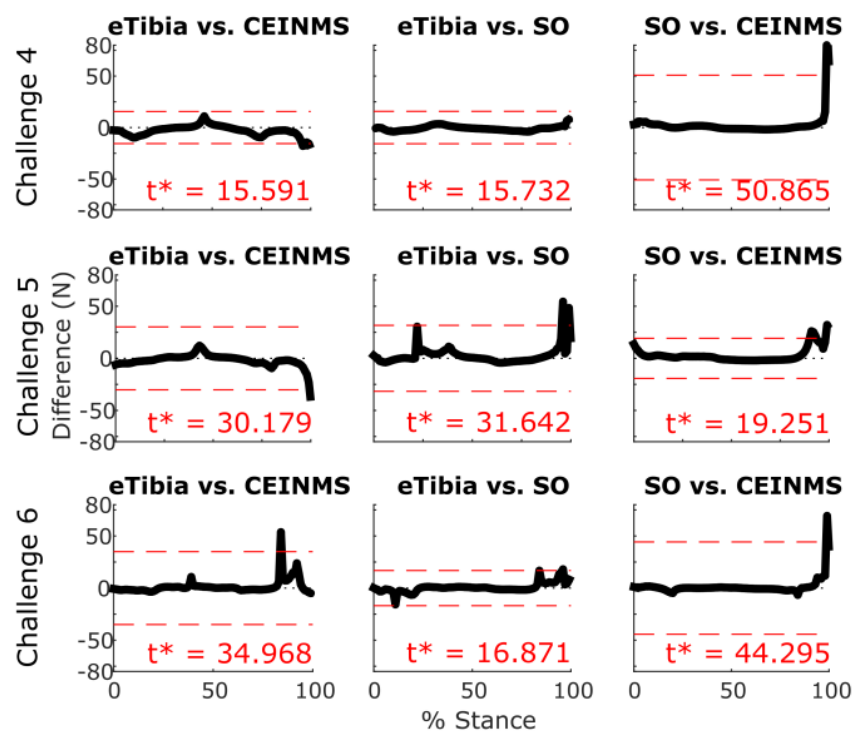

Fig. 5. SnPM results comparing the eTibia measurement to CEINMS (column one), the eTibia measurement to SO (column 2), and SO to CEINMS (column three). Significance is set at $\alpha=0.125$ and all suprathreshold clusters have $\mathrm{p}=0.125$.

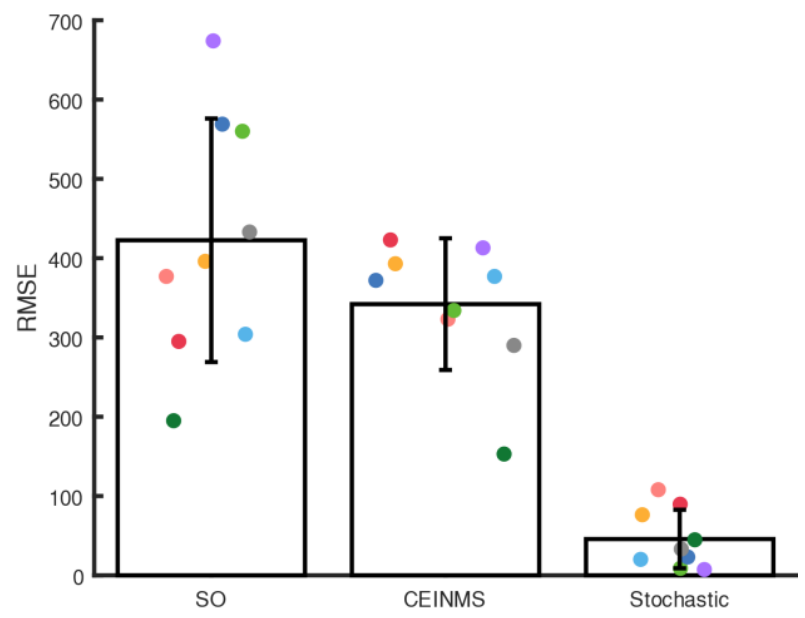

Fig. 6. RMSE mean and standard deviation in estimation of total knee joint loading for SO, CEINMS, and the best fit stochastic solutions. Colors indicate separate trials.

To assess the plausibility of muscle recruitment patterns, we compared the estimated forces of each of the muscles which contribute to knee loading for each method to each other (Fig. 7-9). In all three challenge datasets, the SO solution estimated the medial gastrocnemius force to be 2.75-3.6 times more than the CEINMS solutions. Using the stochastic method, for challenges 4 and 5, where the vastus medialis was constrained to EMG measures, there were no solutions where the rectus femoris activated to one half of its maximum possible force.

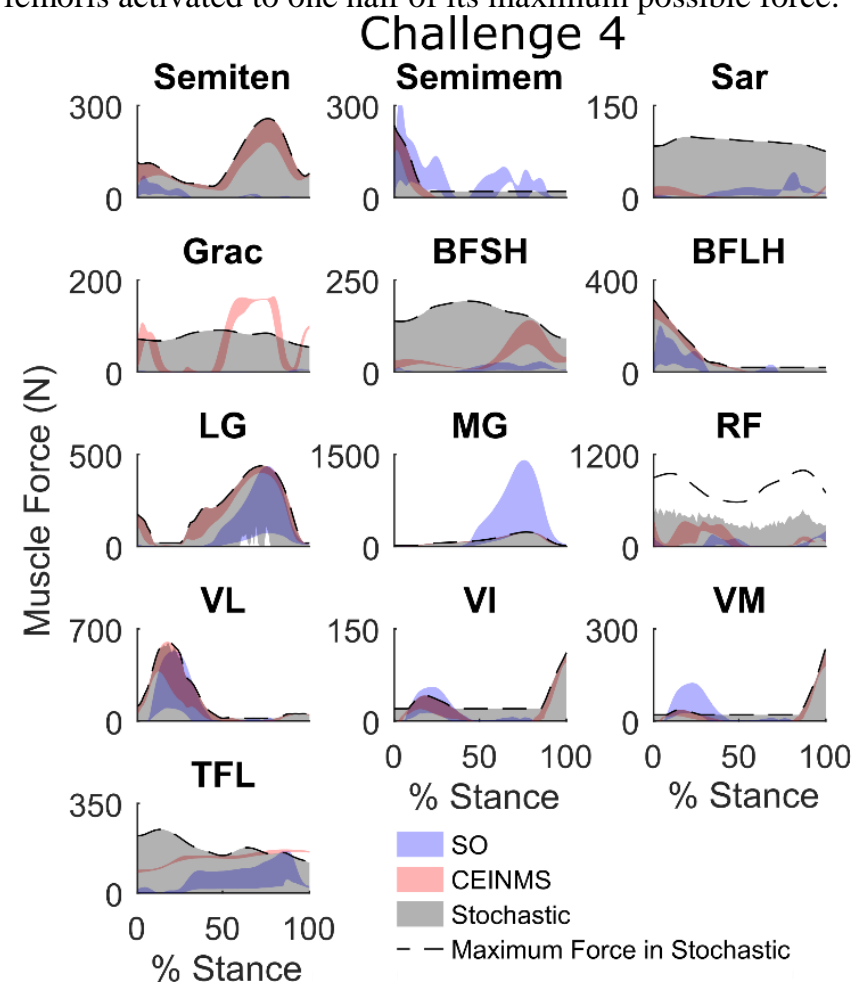

Fig. 6. Muscle forces (N) contributing to joint loading for SO $($ mean \pm SD), CEINMS (mean $\pm \mathrm{SD}$ ), the stochastic method (range) for challenge 4. The muscles presented are: Semiten = semitendinosus; Semimem = semimembranosus; Sar = Sartorius; Grac $=$ gracilis; $\mathrm{BFSH}=$ biceps femoris short head; BFLH = biceps femoris long head; $\mathrm{LG}=$ lateral gastrocnemius; $\mathrm{MG}=$ medial gastrocnemius; $\mathrm{RF}=$ rectus femoris; $\mathrm{VL}=$ vastus lateralis; $\mathrm{VI}=$ vastus intermedialis; $\mathrm{VM}=$ vastus medialis; and TFL = tensor fascia latae.

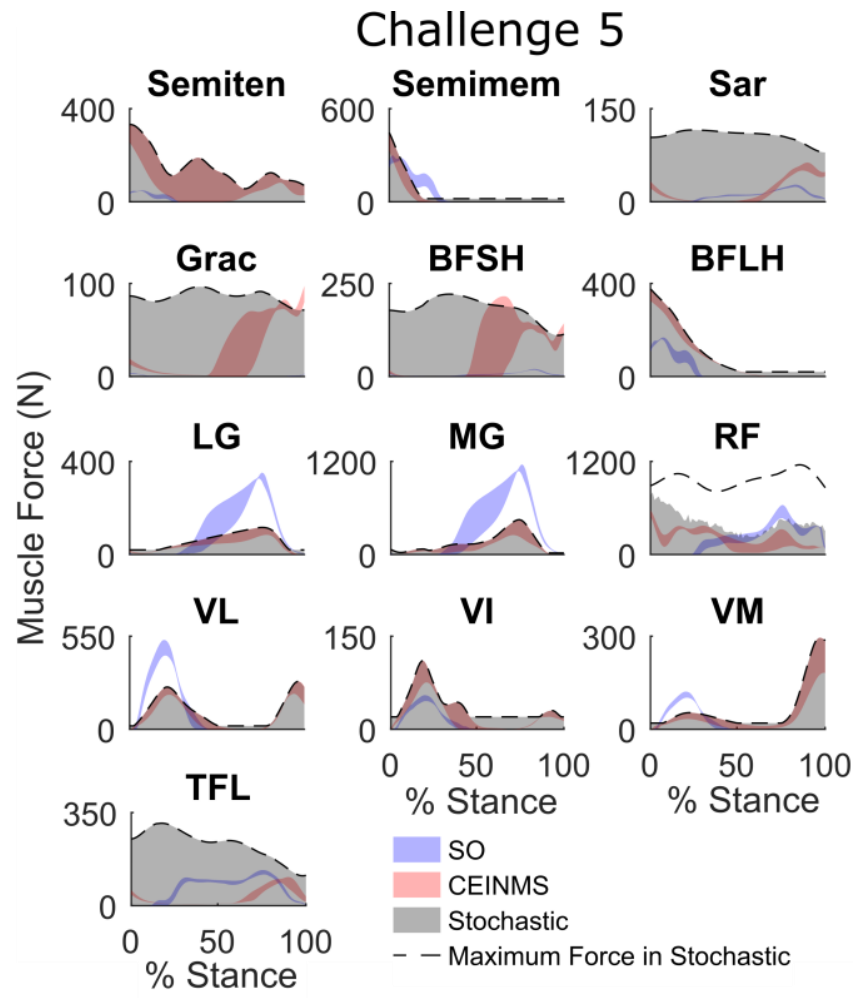

Fig. 7. Muscle forces $(\mathrm{N})$ contributing to joint loading for SO 
(mean $\pm \mathrm{SD}$ ), CEINMS (mean $\pm \mathrm{SD})$, the stochastic method (range) for challenge 5 .

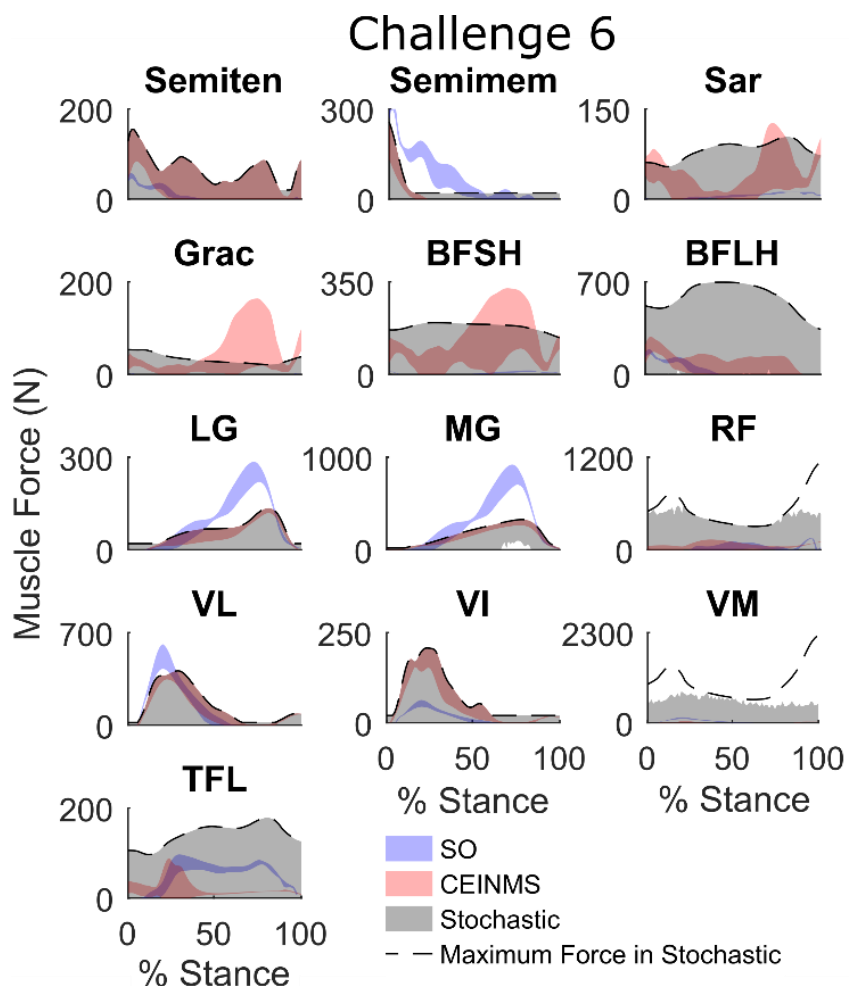

Fig. 8. Muscle forces $(\mathrm{N})$ contributing to joint loading for $\mathrm{SO}$ $($ mean $\pm \mathrm{SD}$ ), CEINMS (mean $\pm \mathrm{SD}$ ), the stochastic method (range) for challenge 6 .

Each method required varying levels of computational resources. SO solutions required seconds to minutes to solve on a desktop PC, whereas the CEINMS calibration step required 25.8 hours with 201,750 evaluations per calibration. All models were calibrated $(3$ subjects $\times 24$ objective functions) simultaneously in 110.5 hours on a desktop HPC (Intel Xeon E52630v4 $2.20 \mathrm{GHz}, 20$ cores). Following calibration, trials were solved in less than one minute each. The stochastic method was implemented on the Phoenix HPC, where eight cores were used to calculate muscle combinations in parallel, requiring 2.5 hours to solve for 93 frames and generating up to $30 \mathrm{~GB}$ of solutions per trial.

\section{DISCUSSION}

This study found that the use of EMG information increases the accuracy of knee loading prediction. A single objective function was identified for calibration of EMG-informed models, which resulted in improved estimates of knee loading when compared to SO. Using an EMG-informed stochastic method, the variation in total knee loading was estimated by investigating the uncertainty in muscle forces.

We identified an optimal objective function for calibrating model neuromuscular parameters to use in EMG-informed modelling. Incorporation of the model estimated load for the joint of interest in calibration has previously been shown to improve estimates of loading at the knee [17] and the hip [14]. Here, we found that by minimizing a combination of the model predicted hip, knee, and ankle loading resulted in the best estimate of knee loading when compared to measured data. The resulting calibrated model, used in combination with an EMGassisted method, also outperformed $\mathrm{SO}$ for multiple individuals, in terms of knee joint contact force prediction accuracy.

Determining the error associated with musculoskeletal simulations was made possible through direct comparison with measured joint loads. Using SO, the second peak loading was overestimated. This was more pronounced in the data from challenge 5, where a peak error of $1432 \mathrm{~N}$ was obtained in one trial. This overestimation was consistent with previous studies reporting on similar datasets $[35,36]$. It is likely that the optimization task preferentially activated the medial gastrocnemius to plantar flex the ankle due to its maximum isometric force. The result of preferentially activating the medial gastrocnemius is an increase in second peak loading at the knee. The EMG-informed approach predicted, on average, more accurate knee contact forces when compared to SO. There was some error in joint loading during initial contact, but estimates of second peak loading were closer to the loads measured.

The directional components of the loads for both SO and CEINMS were different to those measured in both the A-P and $\mathrm{M}-\mathrm{L}$ directions. The results of the stochastic method show that the directional components of joint load could not be estimated based only on the variations in muscle forces. Previous studies using multiple DOF knees have shown good estimates of M-L and A-P loading [37, 38]. While a single DOF planar knee, as used here, is sufficient to obtain estimates of total load, it is unable to accurately estimate the directional components of loading.

The muscle forces generated in the CEINMS solution are different to those from the SO solution. While some larger muscles (vastus and gastrocnemii) show similar activation patterns, weaker muscles show distinctly different activation patterns (biceps femoris, gracilis, sartorius) to generate the same external moments. When comparing muscles which were driven using EMG, the CEINMS solutions used less medial gastrocnemius activation, and instead used other, smaller muscles to drive motion. The stochastic method shows that, using the CEINMS solutions to constrain driven muscle solutions, the constraints on the muscles used for knee flexion (semiten, semimem, biceps femoris, gastrocnemii) limited the maximum knee extensor muscle forces (vastus, rectus femoris) through maintenance of external moment balance.

This study showed, through application, that the stochastic method of muscle recruitment employed can be generalized and applied to multiple individuals. This was previously discussed as a limitation by Martelli et al. [15], where the generalizability of the method was unknown. The use of the stochastic method in this study allowed for further interrogation of muscle recruitment strategies, showing that modeling the knee joint using a single DOF might be insufficient to appropriately estimating the directional component of loading. The stochastic approach allows for investigation into the range of possible muscle recruitment, providing a solution space of joint loadings rather than a single value. This method may be further perturbed to include selective muscle weakness, or to account for error in data collection procedures by modelling the measured parameters as stochastic parameters surrounding the recorded value. 
Using statistical shape models to scale musculoskeletal anatomy reduces error in anatomical definitions by up to $82 \%$ [39], and in kinematic reconstruction when compared to linear scaling $\left(\mathrm{R}^{2}\right.$ range from $0.724-0.997$ for linear scaling, and 0.994-1.000 for statistical shape scaling) [40]. These methods, however, do not account for geometric variance of knee, which has a large effect on reconstructed tibiofemoral motion and subsequent measures of muscle function [41]. This geometric variance also includes differences in muscle insertion locations, directions, and moment arms, which we were unable to account for using statistical shape modelling. These data all require medical imaging, which is available for the Grand Challenge datasets at the knee, but is not typically available for people undergoing gait analysis.

This study was limited by the amount of available data. Constraining this investigation to only static and over ground walking trials meant only five or six trials were available for each challenge. Further, in challenges 4 and 5, some recordings were cropped to within 55 frames before the initial heel strike. To process EMG data and run CEINMS, 75 frames of data are required. Therefore, to run CEINMS, additional frames were added to the beginning of the trials by taking the first $\mathrm{n}$ frames of data, reflecting them, and appending them to the beginning of the trial. Due to the initial feed-forward control implemented in CEINMS, there were higher estimations of joint loading at the start of trials, as the external dynamics were partially from a foot contact event before the true foot contact event, requiring muscles to be active.

We used two walking trials from each participant for calibrating the CEINMS models and did not include them in the final analysis as they may have biased the results. This resulted in three trials for comparison for each participant, which is insufficient for using statistical parametric mapping. Even when using statistical non-parametric mapping, the smallest significance ( $\mathrm{p}$ value) that can be detected is $1 / 2^{\text {ntrials }}$, which for three trials is 0.125. Additionally, including a wide repertoire of motor tasks for the NMS model calibration improved estimated of joint contact forces at the shoulder, compared to calibration that used a single task [42]. It is plausible that these same observations could extend to the knee joint, and that including motor tasks other than walking in the calibration might further improve estimates of knee contact forces.

There was an inconsistency between the hip adduction moments generated in the CEINMS solution and those calculated using inverse dynamics. The gluteus medius typically drives the hip adduction moment, but because the available EMG data was noisy, we decided to exclude it from the simulation. The gluteus maximus (which was driven using EMG) was employed to drive hip motion, but was unable to balance both the flexion the adduction, resulting in the inconsistency identified. This was observed across all calibration functions, suggesting that, when using an EMG informed approach, it is important to capture the activation of the gluteus medius muscles.

When using the stochastic modelling method, we assumed that the muscle forces which were not driven using EMG in CEINMS could only produce half of their maximum possible force. This biased the solution space towards lower estimated joint loads. While this did reduce the solution space to include the measured joint loading, it meant that other solutions were not able to be considered in the sampling.

Finally, as this study only investigated the methods for three individuals, it would be useful to further study the use of the generated CEINMS calibration function on more participants to assess its efficacy in estimating knee joint loading. The availability of additional open access datasets, such as the Grand Challenge [2] and the CAMS knee project [3], might allow for future additional validation of these methods for estimating knee joint loading.

\section{CONCLUSION}

The inclusion of subject-specific muscle activation measures using surface EMG improved estimates of knee joint loading when compared to static optimization. There were deficiencies in estimating the directional components of loading, however the stochastic method employed showed that this is not due to errors in muscle recruitment modelling. Our results show that uncertainty in muscle forces can account for total loading and it is recommended that, where possible, EMG measurements should be included to estimate knee joint loading.

\section{ACKNOWLEDGEMENT}

This work was supported with supercomputing resources provided by the Phoenix HPC service at the University of Adelaide.

[1] D. J. Saxby, B. A. Killen, C. Pizzolato, C. P. Carty, L. E. Diamond, L. Modenese, J. Fernandez, G. Davico, M. Barzan, G. Lenton, S. B. Da Luz, E. Suwarganda, D. Devaprakash, R. K. Korhonen, J. A. Alderson, T. F. Besier, R. S. Barrett, and D. G. Lloyd, "Machine learning methods to support personalized neuromusculoskeletal modelling," Biomechanics and Modeling in Mechanobiology, vol. 19, no. 4, pp. 1169-1185, 2020.

[2] B. J. Fregly, T. F. Besier, D. G. Lloyd, S. L. Delp, S. A. Banks, M. G. Pandy, and D. D. D'Lima, "Grand challenge competition to predict in vivo knee loads," J Orthop Res, vol. 30, no. 4, pp. 503-13, Apr, 2012.

[3] W. R. Taylor, P. Schütz, G. Bergmann, R. List, B. Postolka, M. Hitz, J. Dymke, P. Damm, G. Duda, H. Gerber, V. Schwachmeyer, S. H. Hosseini Nasab, A. Trepczynski, and I. Kutzner, "A comprehensive assessment of the musculoskeletal system: The CAMS-Knee data set," Journal of Biomechanics, vol. 65, pp. 32-39, 2017/12/08/, 2017.

[4] B. A. Killen, A. Falisse, F. De Groote, and I. Jonkers, "In SilicoEnhanced Treatment and Rehabilitation Planning for Patients with Musculoskeletal Disorders: Can Musculoskeletal Modelling and Dynamic Simulations Really Impact Current Clinical Practice?," Applied Sciences, vol. 10, no. 20, 2020.

[5] T. S. Buchanan, D. G. Lloyd, K. Manal, and T. F. Besier, "Neuromusculoskeletal Modeling: Estimation of Muscle Forces and Joint Moments and Movements From Measurements of Neural Command," Journal of applied biomechanics, vol. 20, no. 4, pp. 367-395, 2004.

[6] F. C. Anderson, and M. G. Pandy, "Static and dynamic optimization solutions for gait are practically equivalent," Journal of Biomechanics, vol. 34, no. 2, pp. 153-161, 2001.

[7] S. Martelli, D. Calvetti, E. Somersalo, M. Viceconti, and F. Taddei, "Computational tools for calculating alternative muscle force patterns during motion: A comparison of possible solutions," vol. 46, no. 12, pp. 2097-2100, 2013.

[8] G. E. Loeb, “Optimal isn't good enough," vol. 106, no. 11-12, pp. 757-765, 2012.

[9] S. Martelli, F. Taddei, A. Cappello, S. Van Sint Jan, A. Leardini, 
and M. Viceconti, "Effect of sub-optimal neuromotor control on the hip joint load during level walking," Journal of Biomechanics, vol. 44, no. 9, pp. 1716-1721, 2011.

[10] R. D. Crowninshield, and R. A. Brand, "A physiologically based criterion of muscle force prediction in locomotion," Journal of Biomechanics, vol. 14, no. 11, pp. 793-801, 1981.

[11] S. L. Delp, F. C. Anderson, A. S. Arnold, P. Loan, A. Habib, C. T. John, E. Guendelman, and D. G. Thelen, "OpenSim: Open-Source Software to Create and Analyze Dynamic Simulations of Movement," IEEE Transactions on Biomedical Engineering, vol. 54, no. 11, pp. 1940-1950, 2007.

[12] C. Pizzolato, D. G. Lloyd, M. Sartori, E. Ceseracciu, T. F. Besier, B. J. Fregly, and M. Reggiani, "CEINMS: A toolbox to investigate the influence of different neural control solutions on the prediction of muscle excitation and joint moments during dynamic motor tasks," Journal of biomechanics, vol. 48, no. 14, pp. 3929-3936, 2015.

[13] D. G. Lloyd, and T. F. Besier, "An EMG-driven musculoskeletal model to estimate muscle forces and knee joint moments in vivo," Journal of Biomechanics, vol. 36, no. 6, pp. 765-776, 2003/06/01/, 2003.

[14] H. X. Hoang, L. E. Diamond, D. G. Lloyd, and C. Pizzolato, "A calibrated EMG-informed neuromusculoskeletal model can appropriately account for muscle co-contraction in the estimation of hip joint contact forces in people with hip osteoarthritis," Journal of Biomechanics, vol. 83, pp. 134-142, 2019.

[15] S. Martelli, D. Calvetti, E. Somersalo, and M. Viceconti, "Stochastic modelling of muscle recruitment during activity," Interface focus, vol. 5, no. 2, pp. 20140094-20140094, 2015.

[16] K. Manal, and T. S. Buchanan, "An Electromyogram-Driven Musculoskeletal Model of the Knee to Predict in Vivo Joint Contact Forces During Normal and Novel Gait Patterns," Journal of Biomechanical Engineering, vol. 135, no. 2, pp. 021014, 2013.

[17] P. Gerus, M. Sartori, T. F. Besier, B. J. Fregly, S. L. Delp, S. A Banks, M. G. Pandy, D. D. D'Lima, and D. G. Lloyd, "Subjectspecific knee joint geometry improves predictions of medial tibiofemoral contact forces," Journal of Biomechanics, vol. 46, no. 16, pp. 2778-2786, 2013/11/15/, 2013.

[18] A. Kian, C. Pizzolato, M. Halaki, K. Ginn, D. Lloyd, D. Reed, and D. Ackland, "Static optimization underestimates antagonist muscle activity at the glenohumeral joint: A musculoskeletal modeling study," Journal of Biomechanics, vol. 97, pp. 109348, 2019/12/03/, 2019.

[19] D. G. Lloyd, and T. S. Buchanan, "A model of load sharing between muscles and soft tissues at the human knee during static tasks," $J$ Biomech Eng, vol. 118, no. 3, pp. 367-76, Aug, 1996.

[20] G. Davico, C. Pizzolato, D. G. Lloyd, S. J. Obst, H. P. J. Walsh, and C. P. Carty, "Increasing level of neuromusculoskeletal model personalisation to investigate joint contact forces in cerebral palsy: A twin case study," Clinical Biomechanics, vol. 72, pp. 141-149, 2020/02/01/, 2020.

[21] B. C. van Veen, C. Mazza, and M. Viceconti, "The Uncontrolled Manifold Theory Could Explain Part of the Inter-Trial Variability of Knee Contact Force During Level Walking," IEEE Trans Neural Syst Rehabil Eng, vol. 28, no. 8, pp. 1800-1807, Aug, 2020.

[22] A. Mantoan, C. Pizzolato, M. Sartori, Z. Sawacha, C. Cobelli, and M. Reggiani, "MOtoNMS: A MATLAB toolbox to process motion data for neuromusculoskeletal modeling and simulation," Source Code for Biology and Medicine, vol. 10, no. 1, pp. 12, November 16, 2015.

[23] J. Zhang, H. Sorby, J. Clement, C. D. L. Thomas, P. Hunter, P. Nielsen, D. Lloyd, M. Taylor, and T. Besier, "The MAP Client: User-Friendly Musculoskeletal Modelling Workflows." pp. 182192.

[24] J. Zhang, D. Malcolm, J. Hislop-Jambrich, C. D. L. Thomas, and P. M. F. Nielsen, "An anatomical region-based statistical shape model of the human femur," Computer Methods in Biomechanics and Biomedical Engineering: Imaging \& Visualization, vol. 2, no. 3, pp. 176-185, 2014/07/03, 2014.

[25] P. de Leva, "Adjustments to Zatsiorsky-Seluyanov's segment inertia parameters," Journal of Biomechanics, vol. 29, no. 9, pp. 12231230, 1996/09/01/, 1996.

[26] C. R. Winby, D. G. Lloyd, and T. B. Kirk, "Evaluation of different analytical methods for subject-specific scaling of musculotendon parameters," Journal of Biomechanics, vol. 41, no. 8, pp. 1682-
$1688,2008$.

[27] L. Modenese, E. Ceseracciu, M. Reggiani, and D. G. Lloyd, "Estimation of musculotendon parameters for scaled and subject specific musculoskeletal models using an optimization technique," Journal of Biomechanics, vol. 49, no. 2, pp. 141-148, 2016/01/25/, 2016.

[28] G. G. Handsfield, C. H. Meyer, J. M. Hart, M. F. Abel, and S. S. Blemker, "Relationships of 35 lower limb muscles to height and body mass quantified using MRI," Journal of Biomechanics, vol. 47, no. 3, pp. 631-638, 2014.

[29] A. Hill. "The Heat of Shortening and the Dynamic Constants of Muscle."

[30] M. Sartori, M. Reggiani, D. Farina, and D. G. Lloyd, "EMG-Driven Forward-Dynamic Estimation of Muscle Force and Joint Moment about Multiple Degrees of Freedom in the Human Lower Extremity," PLoS ONE, vol. 7, no. 12, pp. e52618, 2012.

[31] M. Sartori, D. Farina, and D. G. Lloyd, "Hybrid neuromusculoskeletal modeling to best track joint moments using a balance between muscle excitations derived from electromyograms and optimization," Journal of Biomechanics, vol. 47, no. 15, pp. 3613-3621, 2014/11/28/, 2014.

[32] D. G. Thelen, "Adjustment of Muscle Mechanics Model Parameters to Simulate Dynamic Contractions in Older Adults," Journal of Biomechanical Engineering, vol. 125, no. 1, pp. 70-77, 2003.

[33] T. C. Pataky, "One-dimensional statistical parametric mapping in Python," vol. 15, no. 3, pp. 295-301, 2012.

[34] T. C. Pataky, J. Vanrenterghem, and M. A. Robinson, "Zero- vs. one-dimensional, parametric vs. non-parametric, and confidence interval vs. hypothesis testing procedures in one-dimensional biomechanical trajectory analysis," Journal of Biomechanics, vol. 48, no. 7, pp. 1277-1285, 2015/05/01/, 2015.

[35] B. A. Knarr, and J. S. Higginson, "Practical approach to subjectspecific estimation of knee joint contact force," Journal of Biomechanics, vol. 48, no. 11, pp. 2897-2902, 2015/08/20/, 2015.

[36] Z. Imani Nejad, K. Khalili, S. H. Hosseini Nasab, P. Schütz, P. Damm, A. Trepczynski, W. R. Taylor, and C. R. Smith, "The Capacity of Generic Musculoskeletal Simulations to Predict Knee Joint Loading Using the CAMS-Knee Datasets," Annals of Biomedical Engineering, vol. 48, no. 4, pp. 1430-1440, 2020.

[37] M. W. Hast, and S. J. Piazza, "Dual-Joint Modeling for Estimation of Total Knee Replacement Contact Forces During Locomotion," Journal of Biomechanical Engineering, vol. 135, no. 2, pp. 021013 , 2013.

[38] A. Esrafilian, L. Stenroth, M. E. Mononen, P. Tanska, S. V. Rossom, D. G. Lloyd, I. Jonkers, and R. K. Korhonen, "12 Degrees of Freedom Muscle Force Driven Fibril-Reinforced Poroviscoelastic Finite Element Model of the Knee Joint," IEEE Transactions on Neural Systems and Rehabilitation Engineering, vol. 29, pp. 123133, 2021.

[39] J. Bahl, J. Zhang, B. Killen, M. Taylor, L. Solomon, J. Arnold, D. Lloyd, T. Besier, and D. Thewlis, "Statistical shape modelling versus linear scaling: Effects on predictions of hip joint centre location and muscle moment arms in people with hip osteoarthritis," Journal of Biomechanics, vol. 85, 2019.

[40] D. Bakke, and T. Besier, "Shape model constrained scaling improves repeatability of gait data," Journal of Biomechanics, vol. 107, pp. 109838, 2020.

[41] S. Martelli, N. Sancisi, M. Conconi, M. G. Pandy, M. E. Kersh, V. Parenti-Castelli, and K. J. Reynolds, "The relationship between tibiofemoral geometry and musculoskeletal function during normal activity," Gait \& Posture, vol. 80, pp. 374-382, 2020/07/01/, 2020.

[42] A. Kian, C. Pizzolato, M. Halaki, K. Ginn, D. Lloyd, D. Reed, and D. Ackland, "The effectiveness of emg-driven neuromusculoskeletal model calibration is task dependent," Journal of Biomechanics, pp. 110698, 2021/08/25/, 2021. 